International Journal of Pure and Applied Mathematics

Volume 95 No. 4 2014, 623-628

ISSN: 1311-8080 (printed version); ISSN: 1314-3395 (on-line version)

url: http://www.ijpam.eu

doi: http://dx.doi.org/10.12732/ijpam.v95i4.15

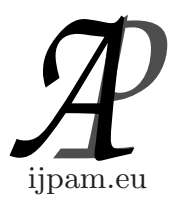

\title{
ON THE NON-DEFECTIVITY AND THE GENERIC $k$-IDENTIFIABILITY FOR SEGRE EMBEDDINGS OF PRODUCTS OF PROJECTIVE VARIETIES
}

\author{
E. Ballico \\ Department of Mathematics \\ University of Trento \\ 38123 Povo (Trento) - Via Sommarive, 14, ITALY
}

\begin{abstract}
We show that recent works of Bocci, Chiantini, Ottaviani and others on the dimension of the secant varieties and their generic identifiability may be translated from Segre embedding of multiprojective spaces to the composition of embeddings $X_{i} \subset \mathbb{P}^{r_{\mathrm{i}}}, 1 \leq i \leq s$, into the Segre embedding of $\mathbb{P}^{r_{1}} \times 1 \cdots \times \mathbb{P}^{r_{\mathrm{s}}}$.
\end{abstract}

AMS Subject Classification: 14N05, 14A

Key Words: Segre variety, secant variety

\section{Introduction}

The aim of this short note is to observe that methods (and even explicit lemmas) from [3], [8] and [4] may be framed in a more general setting. In this setting we do not even need to reproduce the proofs, because they are in the quoted papers. For any subset $A$ of a projective space let $\langle A\rangle$ denote the linear span on $A$. For any integral variety $X \subset \mathbb{P}^{r}$ let let $\sigma_{k}(X)$ denote the closure in $\mathbb{P}^{r}$ of the union of all linear spaces $\langle S\rangle$ with $S \subset X$ and $\sharp(S)=k$ (we will call it the k-secant variety of $X$, but many mathematicians call it the $(k-1)$ th secant variety of $X)$. The set $\sigma_{k}(X)$ is an integral variety of dimension

Received: July 27, 2014

(c) 2014 Academic Publications, Ltd. url: www.acadpubl.eu 
$\leq \min \{r, k(\operatorname{dim}(X)+1)-1\}$. The integer is called the expected dimension of $\sigma_{k}(X)$. Often it is interesting to know if $\sigma_{k}(X)$ has the expected dimension. Often if is very interesting to know if for a general $P \in \sigma_{k}(X)$ there is a unique $S \subset X$ with $\sharp(S)=k$ and $P \in\langle S\rangle$. In this case $X$ is said to be $(k-1)$ identifiable ([6], [7], [3], [8], [4]). This is very important in the case in with $X$ is a product of $s$ projective spaces embedded using the Segre embedding, because it corresponds to tensors $([11])$ and uniqueness guarantees that any solution, $S$, you find for a general tensor with tborder rank $k$ is the only solution. The tools developed in [3] and [4] work for products of varieties which are not projective spaces (as written in several intermediate lemmas by the authors of the quoted papers) and we want to explicitly remark here this fact, because it dramatically improve the range of applications . Moreover, any improvement of the results for the case of multiprojective spaces immediately gives better upper bounds on $k$ for which $X$ is $(k-1)$-identifiable. We fix an integer $k$ and we always work in a range in which $r>k(\operatorname{dim}(X)+1)-1$.

The papers [8] and [4] (in particular [4], ) gives better bounds for the integer $k$, but there are some technical problems to use it in our set-up and hence we prefer to work with the notion of weak defectivity as in [3]. As explained in [?] and [7] if $X \subset \mathbb{P}^{r}$ is not weakly $(k-1)$-defective and $k(\operatorname{dim}(X)+1)-1$, then it is $(k-1)$-identifiable.

The case $C=\mathbb{P}^{q}$ of the following result is [3], Lemma 3.2; we observe that the proof works in the more general case with no modification, just quoting Lemma 1 instead of [3], Lemma 3.1 (the same lemma with $C=\mathbb{P}^{q}$ ).

Theorem 1. Fix an integer $k>0$. Let $Y \subset \mathbb{P}^{a}$ be an integral and nondegenerate variety such that $\operatorname{dim}\left(\sigma_{k}(Y)\right)=k(\operatorname{dim}(Y)+1)-1$. Let $C \subseteq \mathbb{P}^{q}$, $q>0$, be an integral and non-degenerate variety. Set $X:=Y \times C$ seen as a subvariety of $\mathbb{P}^{a b+a+b}$ using the composition of the inclusion $Y \times C \hookrightarrow \mathbb{P}^{a} \times \mathbb{P}^{b}$ using the Segre embedding $\mathbb{P}^{a} \times \mathbb{P}^{b} \hookrightarrow \mathbb{P}^{a b+a+b}$. Then $\operatorname{dim}\left(\sigma_{k}(X)\right)=k(\operatorname{dim}(X)+$ $1)-1$ and $X$ is $(k-1)$-identifiable, i.e. for a general $P \in \sigma_{k}(X)$ there is a unique $S \subset X$ such that $\sharp(S)=k$ and $P \in\langle S\rangle$.

In many cases one knows that $\operatorname{dim}\left(\sigma_{k}(Y)\right)=k(\operatorname{dim}(Y)+1)-1$ (e.g., if $Y$ is a Veronese embedding of a projective space or if $Y$ is a curve and $a \geq 2 k-1)$.

We now give two quick methods to check that $\operatorname{dim}\left(\sigma_{k}(Y)\right)=k(\operatorname{dim}(Y)+$ 1) - 1 for a variety $Y \subset \mathbb{P}^{a}$, when $C$ is a product of lower dimensional varieties (Propositions 1 and 2).

Proposition 1. Let $X_{i} \subset \mathbb{P}^{r_{i}}, 1 \leq i \leq s$, be integral and non-degenerate varieties. Set $n_{i}:=\operatorname{dim}(X)$ and $X:=X_{1} \times \cdots \times X_{s}$ seen as a subvariety of $\mathbb{P}^{r}$, $r:=-1+\prod_{i=1}^{s}\left(r_{i}+1\right)$, using the composition of the inclusion $X \hookrightarrow \mathbb{P}^{r_{1}} \times \cdots \times \mathbb{P}^{r_{\mathrm{s}}}$ 
and the Segre embedding $\mathbb{P}^{r_{1}} \times \cdots \times \mathbb{P}^{r_{\mathrm{s}}} \hookrightarrow \mathbb{P}^{r}$. Fix an integer $k \geq 2$. See $\mathbb{P}^{n_{1}} \times \cdots \times \mathbb{P}^{n_{\mathrm{s}}}$ embedded in $\mathbb{P}^{n}, n:=-1+\prod_{i=1}^{s}\left(n_{i}+1\right)$ and $\mathbb{P}^{n_{1}} \times \cdots \times \mathbb{P}^{n_{\mathrm{s}-1}}$ embedded in $\mathbb{P}^{\rho}, \rho:=-1+\prod_{i=1}^{s-1}\left(n_{i}+1\right)$

(a) If $\operatorname{dim}\left(\sigma_{k}\left(\mathbb{P}^{n_{1}} \times \cdots \times \mathbb{P}^{n_{\mathrm{s}}}\right)\right)=k\left(n_{1}+\cdots+n_{s}+1\right)-1$, then $\operatorname{dim}\left(\sigma_{k}(X)\right)=$ $k\left(n_{1}+\cdots+n_{s}+1\right)-1$.

(b) If $\operatorname{dim}\left(\sigma_{k}\left(\mathbb{P}^{n_{1}} \times \cdots \times \mathbb{P}^{n_{s-1}}\right)\right)=k\left(n_{1}+\cdots+n_{s-1}+1\right)-1$, then $\operatorname{dim}\left(\sigma_{k}(X)\right)=k\left(n_{1}+\cdots+n_{s}+1\right)-1$ and $X$ is $(k-1)$-identifiable.

Proposition 2. Fix integral and non-degenerate varieties $Y \subset \mathbb{P}^{a}, C \subset \mathbb{P}^{b}$ and an integer $k \geq 2$. Assume $\operatorname{dim}\left(\sigma_{k}(C)\right)=k(\operatorname{dim}(C)+1)-1$. Write $X:=$ $Y \times C$ embedded into $\mathbb{P}^{a b+a+b}$ use the composition of the embedding $Y \times C \hookrightarrow$ $\mathbb{P}^{a} \times \mathbb{P}^{b}$ and the Segre embedding $\mathbb{P}^{a} \times \mathbb{P}^{b} \hookrightarrow \mathbb{P}^{a b+a+b}$. Then $\operatorname{dim}\left(\sigma_{k}(X)\right)=$ $k(\operatorname{dim}(X)+1)-1$.

From [5] and Proposition 1 we get the following corollary.

Corollary 1. Let $C_{i} \subseteq \mathbb{P}^{r_{\mathrm{i}}}, 1 \leq i \leq s$, be integral and non-degenerate curves. Set $X:=C_{1} \times \cdots C_{s}$ seen a a subvariety of $\mathbb{P}^{r}, r:=-1+\prod_{i=1}^{s}\left(r_{i}+1\right)$ using the Segre embedding of $\mathbb{P}^{r_{1}} \times \cdots \times \mathbb{P}^{r_{\mathrm{s}}}$. Fix an integer $k$ such that $2 k-1 \leq 2^{s}$. If $s \geq 5$, then $\sigma_{k}(X)$ has the expected dimension. If $s \geq 6$ and $2 k-1 \leq 2^{s-1}$, then $X$ is $(k-1)$-identifiable.

There are huge lists of cases for which $\operatorname{dim}\left(\sigma_{k}\left(\mathbb{P}^{n_{1}} \times \cdots \times \mathbb{P}^{n_{\mathrm{s}}}\right)\right)=k\left(n_{1}+\right.$ $\left.\cdots+n_{s}+1\right)-1$ (see [1], [8], and the papers which will quote them).

These statements work also for Segre-Veronese embeddings, e.g. taking as $X_{i} \subset \mathbb{P}^{r_{i}}$ a Veronese embedding of $\mathbb{P}^{n_{\mathrm{i}}}$, or in Theorem 1 taking as $C$ a projective space $\mathbb{P}^{m}$ and as $C \subset \mathbb{P}^{q}, q=\left(\begin{array}{c}m+d \\ m\end{array}\right)-1$, a Veronese embedding of degree $\geq 3$ of $C$ (with the 4 exceptions in the Alexander-Hirschowitz theorem). We may also take as $C \subset \mathbb{P}^{q}$ a general linear projection of the Veronese embedding of $\mathbb{P}^{m}$ if $q \geq k(\operatorname{dim}(C)+1)-1$.

We work over an algebraically closed field $\mathbb{K}$ such that $\operatorname{char}(\mathbb{K})=0$.

\section{Outline of the Proofs}

For any quasi-projective variety $X$ and $P \in X_{\text {reg }}$ let $(2 P, X)$ denote the first infinitesimal neighborhood of $P$ in $X$, i.e. the closed subscheme of $X$ with $\left(\mathcal{I}_{P}\right)^{2}$ as its ideal sheaf.

Lemma 1. Fix positive integers $a, q, k$, an integral and non-degenerate variety $C \subseteq \mathbb{P}^{q}$, and any $k$ linear subspaces $H_{1}, \ldots, H_{k} \subseteq \mathbb{P}^{a}$ such that $\operatorname{dim}\left(\left\langle H_{1} \cup\right.\right.$ $\left.\left.\cdots \cup H_{k}\right\rangle\right)<\sum_{i=1}^{k} \operatorname{dim}\left(H_{i}\right)+k-1$. Fix general $P_{1}, \ldots, P_{k} \in C$. See $\mathbb{P}^{a} \times C$ 
as a subvariety of $\mathbb{P}^{a q+a+q}$ using the Segre embedding. Then $\operatorname{dim}\left(\left\langle H_{1} \times\left\{P_{1}\right\} \cup\right.\right.$ $\left.\left.\cdots \cup H_{k} \times\left\{P_{k}\right\}\right\rangle\right)>\operatorname{dim}\left(\left\langle H_{1} \cup \cdots \cup H_{k}\right\rangle\right)$

Proof. Up to a permutation of the indices we may assume $H_{1} \cap\left\langle H_{2} \cup \cdots \cup\right.$ $\left.H_{k}\right\rangle \neq \emptyset$. As observed in [3] the inequality $\operatorname{dim}\left(\left\langle H_{1} \times\left\{P_{1}\right\} \cup \cdots \cup H_{k} \times\left\{P_{k}\right\}\right\rangle\right)>$ $\operatorname{dim}\left(\left\langle H_{1} \cup \cdots \cup H_{k}\right\rangle\right)$ holds if we take any $P_{1}, P_{2}$ with $P_{1} \neq P_{2}$ and $P_{j}=P_{2}$ for all $j>2$ by the structure of the Segre embedding. Hence it holds for a general $\left(P_{1}, \ldots, P_{k}\right) \in C^{k}$.

Lemma 2. Fix integral and non-degenerate varieties $Y \subseteq \mathbb{P}^{a}, C \subset \mathbb{P}^{b}$ and an integer $k \geq 2$. Assume $\operatorname{dim}\left(\sigma_{k}(C)\right)=k(\operatorname{dim}(C)+1)-1$. See $\mathbb{P}^{a} \times \mathbb{P}^{b}$ as a subvariety of $\mathbb{P}^{a b+a+b}$ using the Segre embedding. Fix $k$ general points $Q_{1}, \ldots, Q_{k}$ of $Y$ and let $M_{i}, i=1, \ldots, k$, be the linear span of the biprojective space $\mathbb{P}^{a} \times T_{Q_{\mathrm{i}}} C$ in $\mathbb{P}^{a b+a+b}$. Each $M_{i}$ has dimension $a \cdot \operatorname{dim}(C)+a+\operatorname{dim}(C)$ and they are linearly independent in $\mathbb{P}^{a b+a+b}$.

Proof. This is a trivial consequence of the definition of the Segre embedding, since the linear spaces $T_{Q_{\mathrm{i}}} C, i=1, \ldots, k$, are linearly independent.

Proof of Proposition 2: Set $e:=\operatorname{dim}(Y)$ and $f:=\operatorname{dim}(C)$. By the characteristic free part of Terracini's lemma ([2], Corollary 1.10) and the semicontinuity theorem for cohomology ([10], III.12.8), it is sufficient to find $S \subset X_{\text {reg }}$ such that $\sharp(S)=k$ and $\operatorname{dim}\left(\left\langle\cup_{P \in S} T_{P} X\right\rangle\right)=k(e+f+1)-1$. Fix any $A \subset Y_{\text {reg such }}$ that $\sharp(A)=k$. By Lemma 2 there is an injective map $u: A \rightarrow C_{\text {reg }}$ such that the set $T_{Q} Y \times T_{u(Q)} C, Q \in A$, spans linearly independent subspaces; here each $T_{Q} Y \times T_{u(Q)} C$ is the Segre product of the $e$-dimensional projective space $T_{Q} Y$ and an $f$-dimensional projective space $T_{u(Q)} C$ and hence this Segre product spans a linear space of dimension $e f+e+f$; Lemma 2 implies that these $k$ linear spaces span a linear space of dimension $k(e f+e+f+1)-1$. Fix $Q \in A$ and set $P_{Q}:=(Q, u(Q)) \in X$. Set $S:=\cup_{Q \in A} P_{Q}$. We have $\sharp(S)=k$. For each $P=(Q, u(Q)) \in S$ the tangent space $T_{P} X \subset \mathbb{P}^{a b+a+b}$ is a linear subspace of $\left\langle T_{Q} Y \times T_{u(Q)} C\right\rangle$, because the zero-dimensional scheme $(2 P, X)$ is contained in the scheme $(2 Q, Y) \times(2 u(Q), C)$. Hence these $k$ tangent spaces to points of $X_{\text {reg }}$ are linearly independent.

Proof of Proposition 1: Set $W:=\mathbb{P}^{n_{1}} \times \cdots \times \mathbb{P}^{n_{\mathrm{s}}}$ and $W^{\prime}:=\mathbb{P}^{r_{1}} \times \cdots \times \mathbb{P}^{r_{\mathrm{s}}}$. Taking a general linear projection we get a finite separable map $X_{i} \rightarrow \mathbb{P}^{n_{\mathrm{i}}}$. The map $\pi=\left(\pi_{1}, \ldots, \pi_{s}\right): X \rightarrow W$ is finite and separable. Fix a general $S \subset X$ such that $\sharp(S)=k$. The set $\pi(S)$ is a general subset of $W$. Since

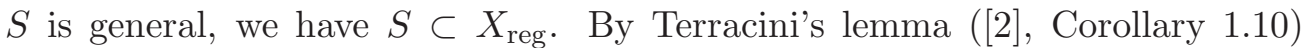
and the assumption of $\sigma_{k}(W)$ the linear span of of $(2 \pi(S), W)$ has dimension 
$k\left(n_{1}+\cdots+n_{s}\right)+k-1$. Since $W \subset \mathbb{P}^{n}$ is a linear projection of $W^{\prime} \subset \mathbb{P}^{r}$ from a linear subspace intersecting $W^{\prime}$, but disjoint from $X$ and hence from $S$, we get that $\langle(2 S, W)\rangle$ has dimension $k\left(n_{1}+\cdots+n_{s}\right)+k-1$. Hence $\sigma_{k}(X)$ has dimension $k\left(n_{1}+\cdots+n_{s}\right)+k-1$, concluding the proof of part (a).

Part (b) follows from part (a) applied to the first $s-1$ factors of $X$ and Theorem 1 applied to $Y=X_{1} \times \cdots X_{s-1}$ and $C:=X_{s} \subset \mathbb{P}^{r_{s}}$.

Remark 1. Take $X$ as in Theorem 1. Fix general $Q_{1}, \ldots, Q_{k} \in X$ and a general hyperplane $H \subset \mathbb{P}^{r}$ containing the tangent spaces $T_{Q_{\mathrm{i}}} X, i=1, \ldots, k$. Let $W \subset X$ be the union of the irreducible components of of the contact locus of $H$ with $X$ and containing one of the points $Q_{1}, \ldots, Q_{k}$. The last 3 sentences of the proof of [3], proof of Lemma 3.2, show that either $W=\left\{Q_{1}, \ldots, Q_{k}\right\}$ (i.e. $X$ is not weakly $(k-1)$-defective) or $W$ is a union of $k$ linear spaces $A_{1}, \ldots, A_{k}$ with the same dimension and with $Q_{i} \in A_{i}, i=1, \ldots, k$. This is often excluded by the geometry of $X$ (e.g. in the set-up of Corollary 1 this is impossible if $r_{i} \geq 2$ for all $i$ ). In these cases no such $W \subset X$ exists, independently of the further restriction that it must be a generic contact locus. Adding this condition many other cases may be excluded, but not all, as the case $s=3, r_{1}=r_{2}=1$, $r_{3}=2, C_{1}=C_{2}=\mathbb{P}^{1}, C_{3}=\mathbb{P}^{2}$, given in [3], Example 2.13, shows.

\section{Acknowledgements}

The author was partially supported by MIUR and GNSAGA of INdAM (Italy).

\section{References}

[1] H. Abo, G. Ottaviani and C. Peterson, Induction for secant varieties of Segre varieties. Trans. Amer. Math. Soc. 361, 767-792 (2009)

[2] B. Ådlandsvik, Joins and higher secant varieties. Math. Scand. 61, 213-222 (1987)

[3] C. Bocci and L. Chiantini, On the identifiability of binary Segre products. J. Algebraic Geom. 22, 1-11 (2013)

[4] C. Bocci, L. Chiantini and G. Ottaviani, Refined methods for the identifiability of tensors, Annali di Matematica DOI 10.1007/s10231-013-0352-8 
[5] M. V. Catalisano, A.V. Geramita and A. Gimigliano, Secant varieties of $\mathbb{P}^{1} \times \cdots \times \mathbb{P}^{1}(n$-times $)$ are not defective for $n \geq 5$. J. Algebraic Geom. 20(2), 295-327 (2011)

[6] L. Chiantini and C. Ciliberto, Weakly defective varieties. Trans. Amer. Math. Soc. 354(1), 151-178 (2002)

[7] L. Chiantini and L., Ciliberto, On the concept of k-secant order of a variety. J. London Math. Soc. 73, 436-454 (2006)

[8] L. Chiantini and G. Ottaviani, On generic identifiability of 3-tensors of small rank. SIAM J. Matrix Anal. Appl. 33(3), 1018-1037 (2012)

[9] R. Elmore, P. Hall and A. Neeman, An application of classical invariant theory to identifiability in nonparametric mixtures. Ann. Inst. Fourier (Grenoble) 55 (2005), no. 1, 1-28.

[10] R. Hartshorne, Algebraic Geometry, Springer-Verlag, Berlin-HeidelbergNew York, 1977.

[11] J. M. Landsberg, Tensors: Geometry and Applications. Graduate Studies in Mathematics, Vol. 128, Amer. Math. Soc. Providence, 2012. 